\title{
6
}

\section{The Feature Interaction Problem in the IN: In search of a global solution}

\author{
Nadir Belarbi \\ University of Versailles - PRiSM Laboratory \\ 45, avenue des Etats Unis- 78000 Versailles - France \\ Tel : +331392543 32,Fax : +33139254057 \\ E-mail:NaBe@prism.uvsq.fr / belarbi@inf.enst.fr

\section{Dominique Gaiti} \\ Center for Telecommunications Research - Columbia University \\ and UPMC - Casier 204 \\ 4, place Jussieu 75252 Paris Cedex 05 - France \\ Tel: +331442738 89, Fax: +33144274837 \\ E-mail: gaiti@ctr.columbia.edu
}

\begin{abstract}
Telecommunication operators are looking for new types of services to be introduced in multimedia networks. It is a good way to speed up the design of such networks and to raise the reliability and the efficiency of the actual telecommunication networks. During the last years, new types of services were introduced by the bell operating companies around the world. The toll free number is a key service that first permitted to realize that the introduction of new services in classical telecommunication networks is a difficult task. Ever more logical concepts and less and less physical attachment are needed to bring up more flexibility in the telecommunication network architecture to be able to take in charge new services. The Intelligent Network (IN) as a distributed system working in real time is particularly complex to design and to manage. The purpose of our article is to give a general overview of the feature interactions problem considered as one of the main problem in the IN.
\end{abstract}




\section{INTRODUCTION}

During the 1980s, the hardware components of a typical 15,000 lines PBX decreased by $45 \%$ (from $55 \%$ of hardware to $30 \%$ ) while the software components increased of $1500 \%$ (from 5 packages to 80) [NT 89].

Today, it seems logical to say that telecommunication networks rely essentially on software. Therefore, problems of such networks are now, in major cases, software problems.

The difficulty for designing and for developing software of such complex distributed systems is an important obstacle in the development of new services in the Intelligent Network (IN). Telecommunication software must proceed in real time and has to be distributed. It is difficult to add new services to an existing system without modifying its operations. Each new service may interact with many existing services, causing an unexpected service behavior or a system failure. This problem is generally called a feature interaction in the area of telecommunication systems [Bowen 89].

Many papers have been published about the design process of a service (from waterfall models to the idea of growing software); many methodologies have been proposed (from the structure analysis and design to the object orientation), and many approaches have been suggested (from drawing pictures to formal methods with mathematical foundations). Tools have been provided for most of the above schemes [Linden 94]. None of these have turned out to be the Silver Bullet they promised to be, may be because most approaches tackle the accidental tasks that arise from software production and not the essence of the conceptual model [Brooks 87], [Harel 92]. It seems obvious to say that there is no global methodology to solve the feature interaction. The results of different research fields are needed to address all facets of the feature interaction problem. These fields include, at the minimum, formal description methods, software engineering, protocol engineering, distributed computing and distributed artificial intelligence [Cameron 93].

The consequence of this situation is that several methodologies try to address the interaction problem at different stages of the service feature life-cycle. We are going to introduce some of the main methodologies involved in the management of the interaction problem.

The remainder of this paper is organized as follows. The next section introduces the feature interaction problem in the IN and the main strategies used for its management. The third section gives first, an overview of the main concepts used by these strategies. Then, we present some of the main solutions corresponding to each strategy. Finally, some remarks conclude the paper. We will not investigate here all the existing methodologies and examine in detail their mechanisms. For more details see [Cameron 94], [Cameron 93], [IEEE 93a], [IEEE 93b], [IEEE 93c], [Bowen 89]. We rather try to focus on the main concepts they use to reach their goals in each kind of strategy. 


\section{THE FEATURE INTERACTION IN THE IN}

\subsection{An introduction}

The feature interaction problem seems to be an inherent characteristic of the IN. Every time a new service is introduced in the IN, there is a high probability that this service process will meet problems within the new environment. The design of new services is a critical task because we cannot anticipate all the possible situations new services will meet during their life time in the IN. The problem is very similar to the problem of designing new software in computer science: a long life cycle is needed to validate the product.

To fulfill customer demands, an increasing proportion of value added services must be provided through the software. This adds significantly to the flexibility of the switching and transmission components but this increases the complexity of the resulting systems. Software design, installation, testing, configuration, and maintenance in a widespread heterogeneous environment are becoming increasingly difficult. With each new service and/or feature, systems become more complex. Those systems are connected in places where no such interconnections were foreseen. It is hard to understand the whole system and to be sure that additions and interconnections will have the desired effect and not another [Linden 94].

Generally three categories of approaches are used to address the problem of feature interactions [Cameron 93] :

- Avoidance;

- Detection;

- Resolution.

These three approaches are complementary. It seems impossible to avoid completely interaction problems even when a correct specification of the services is provided. For that reason, detection and resolution steps generally follow the avoidance step. Detection and resolution steps seem to be related. The detection of an interaction can for instance call a resolution procedure and so on. Many approaches are used in each category, and they can be divided into two main groups: off-line and on-line approaches [Bouma 94]. The first type of approach operates before the process of a service in the IN. The distinction between off-line and on-line approaches is not significant in the avoidance step, but has a strong meaning in the detection and the resolution steps [Bouma 94].

We prefer to talk about the management of an interaction when we use the concept of avoidance, detection and resolution: solving completely an interaction is an optimal situation that can rarely be completed without effects on the process of one or several services.

We notice that few methodologies, dealing with the IN interference problem, address only one category of the mentioned approaches. Detection methodologies for instance try to propose a resolution step for the detected interaction and that seems natural. Avoidance methodologies are generally not well adapted to the resolution techniques. However, in some cases avoidance methodologies can include concepts strongly associated with detection and resolution problems (the Meeting Organizer in [Cross 94]). 
It is also very difficult in some cases to say if a methodology avoids or detects interactions. The general tendency is to have a methodology including both aspects: first a reliable framework based on the experience from the software life cycle and second, a mechanism that permits a controlled management of the service feature operations. It is important to see that some detection methodologies try also to avoid feature interactions, in supplying control mechanisms. The on-line and off-line concepts [Bouma 94] are also strongly correlated in the detection approaches: the simulated environment can be very close to the real IN environment and so can permit the direct use of off-line detection concepts. Thus, a solution can be obtain at the service interactions through the on-line situation. All these common points underline the relativity of the avoidance, detection and resolution categorization. In spite off all, such classification is needed for a best description of the feature interaction problem, during the life cycle of a service. These three different approaches can be combined in different ways for the elaboration of a particular solution, for the management of the interaction problem (Figure 1).

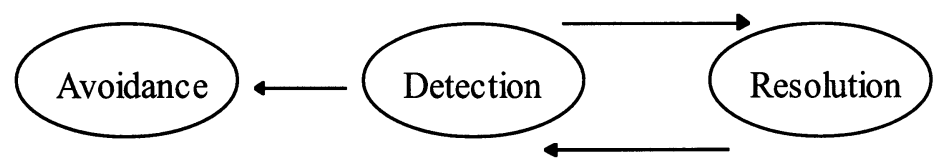

Figure 1 Functionalities of a complete system for the management of interaction problems.

An optimal system for the management of interaction problems can be composed by three components having three different functionalities (Figure 1). We suppose here that the service is at its final life cycle stage, running in an environment close to the IN or directly in the IN.

The service will check at each of its actions if there are no interaction problems. If an interaction problem is detected by the detection component, a resolution strategy is engaged by another specific component until a solution is found. Once this solution has been applied successfully to the resolution of the interaction, it is recorded by the resolution component and the detection component. This new detection information can also be used to avoid situations that could lead to an interaction in the IN.

\subsection{A definition}

A feature interaction problem is generally defined as an unexpected service behavior. The introduction of a new service may cause behavior changes for other services (policy interference). The new service may also behave itself in a different way it was supposed to do. The problem is that this new behavior in the service and their consequences are not wanted and planed. Operators and users can have specific views of feature interactions.

Let us provide with an example of a feature interaction from the user's point of view. We consider the emergency number (911 in the USA) and the three ways calling.

One characteristic of the 911 number is that when the 911 operator answers a call, it could be ended only by this operator. We suppose $\mathrm{A}$ is calling $\mathrm{B}$, and during their conversation 
suddenly A is in an emergency. If B adds the 911 operator as a third party, the properties of the 911 number apply to B's line, but not to A's. Ideally, the properties of the "911" should be apply to A's as well; A can still hang up, and B cannot [Cameron 93].

The feature interaction problem from the operator's point of view can be different and varied. The problem may occur from an incompatibility with the user profile [Mierop 92], an unreachable goal [Faci 94], a logical model unworkable [Gammelgaard 94], an inconsistent system description [Blom 94], a state transition different from the corresponding state signature [Tsang 94] or an updated inconstancy [Cameron 91].

\section{THE MANAGEMENT OF FEATURE INTERACTIONS IN THE IN}

Several approaches are available to solve a feature interaction problem. All these approaches agree with the necessity to downsize the complexity of the system with the use of different architecture models. Modularity and decomposition of the systems in several blocks are key concepts of these methodologies. We remark that all these methodologies tend naturally to use object oriented models and their interesting properties [Mierop 92], [Erradi 92], [Arango 92].

The modularity of a structured system is a natural characteristic of an object oriented system This one may be easily extended and managed. The object model is based on several elements, as the abstraction, the encapsulation, the modularity, and the hierarchy. This provides the way to define a precise responsibility to each object of the system. All these characteristics downsize the complexity of the system and permit to express temporal and space constraints. Using the Case Driven Modeling (UCDM), coming from the object oriented software engineering, seems to be an efficient and helpful technique to design new services [Kimbler 94b].

Generally all the methodologies agree with the necessity to model the system with an architecture based on several levels of abstraction. These levels are related to each other through interfaces that exchange precise data. TINA and INCM are two examples of such architectures [TINA 92], [ITU-T Q120xx, 93]. The IN should take into account heterogeneous equipment that are distributed over its whole structure. For that, IN architecture can follow different existing models like ANSA [APM 91], ODP [ISO/IEC 10746] or CORBA [OMG 94].

Thus, it seems logical to see the IN as a distributed system, composed of several entities, that must work together to achieve several goals. The object is the fundamental entity that constructs the whole IN system. This component can be called application, entity or agent, but it is an object in its role and in its structure. These objects must communicate and exchange information to ensure the operation of the system. The intelligent agent approach, which is an extension of the object concept, tends to permit the modification of the internal structure of these objects and thus to adapt their behavior to the system operations by using the negotiation principle [Griffeth 94].

The use of formal specifications and languages to describe the structure of the modeled system seems to be a mandatory condition to get a correct and coherent description of the system. LOTOS [ISO 8807], SDL [ITU-T Z.100] and ESTELLE [ISO 9074] are some 
examples of such formal languages. LOTOS is strongly used because of its capability to define parallel and synchronizing constraints in the behavior of the modeled process.

The dynamic of the system (the service) is generally modeled by a sequence of different states which takes part of a Finite State Machine (FSM). Each state or a combination of states corresponds to a particular event of the system (service) functioning. The use of a formal framework as a Finite State Machine (FSM) [Hopcroft 79] permits a powerful management of the system with time constraints, using efficient mathematical techniques.

Avoidance and detection schemes can be associated to these mathematical techniques. Constraints [Halpern 90] or logical rules [Gammelgaard 94] [Combes 94] can control the operations of the system and can check the different features of the system. These techniques validate the state transitions and thus detect possible interactions. A similar approach consists to associate to each Service Feature a state transition sequence called a state signature [Tsang 94] or a Feature Profile [Mierop 92]. These approaches can be implemented using a centralized or a distributed knowledge database. A distributed knowledge base permits the association of a specific control entity to each feature: Feature Manager (FM) [Cain 92], [Tsang 94], Feature Interaction Manager (FIM) [Griffeth 94] or User Profile [Mierop 92].

In the resolution step we are free to redesign or to adapt the service to the system (after testing the new process). Redesigning a service is an efficient way to control its behavior and to avoid interaction problems and unspecified operations. But the redesign step is a long and a hard (off-line) technique which is generally experimented in a simulated environment.

Another solution consists to give to the service feature an intelligent and autonomous behavior that permits the negotiation concept [Griffeth 94]. Such intelligent services will be introduced in the $\mathrm{IN}$ to provide an automatic adaptation to the structures and the processes. A negotiation approach will require a certain delay to solve feature interaction problems [Kimbler 94b]. The problem is to know if this negotiation period will not affect the quality of service.

\subsection{Avoidance step}

The schemes to avoid service interaction problems may be provided directly by the platform supporting the service or by the service creation environment. That leads to a service implementation which is intrinsically independent on the interactions [Cameron 93]. Such approaches incorporate open distributed platforms in telecommunications systems to deal with interactions that are qualified of "intrinsic problems in distributed systems" [Cameron 94].

Experiences to validate the restructuring approach to feature interaction, indicate that the feature interaction needs to be viewed at different levels in the system, starting at the highest level with the customer or enterprise point of view and going through various levels [Herbert 93] [Cross 94]. A feature interaction at one level is not necessarily the same feature interaction at another level. Thus, an undetected interaction at one level may involve problems at different levels. The relationship between two levels may be complex and the detection of the interaction quite impossible to find. 
[Linden 94] suggests that there are two ways to reduce the complexity of distributed computing systems and their design. First, it is possible to introduce constraints on the structure of the systems to define clearly the interfaces between the software components and the ways these components may interact. In that case components can be easily analyzed and replaced due to these well-defined interfaces. Second, it is possible to provide a framework to describe the system, the software components and the interactions between them in an independent way of the system design. Principles of separation and substitutability are used to reduce the feature interaction. Separation suggests that two entities (features) have to be completely physically separated. In that manner there is no way for two features to interact (in a positive or a negative manner). Substitutability suggests that software components can be replaced or extended by other components that are compatible.

A similar view is used by [Cross 94] where the entities added to the system are not features, but non-interacting applications. The features of a service may be modified through new components that have to be added in the communication units.

This new paradigm affords an organized range of parameters that includes a choice in the temporal connections between services, a choice in the route to transport the information and a choice in the participants. MORGAN is an implementation of this model with an objectoriented architecture that permits to organize meetings. In this architecture there is a collaboration between the different classes that represent the system. Additional objects include the Meeting Organizer which incorporates the functionality for organizing meetings and a knowledge base which contains the rules to schedule the meetings [Bendeich 93]. MORGAN solves feature interaction at its lowest level by using a matrix.

A knowledge-based representation is also used to create an unambiguous understanding of the requirements of the telephone features [Dankel 94].

Specifications of these requirements, written in an English-based language, are described in a natural language-based system into the knowledge-based representation (requirement capturing system : GATOR). A natural language is used to introduce the information on the interface. An interpreter updates the knowledge base to store this information. The goal of this conversion is to provide less ambiguous written mandatory documents and to update automatically existing requirements documents when new interactions are found.

Other approaches try to extend the existing signaling protocols to prevent interactions [Cameron 93]. Guidelines for a service creation and a service management also take part in those avoidance schemes.

\subsection{Detection}

All interaction problems cannot be solved just by applying a good avoidance methodology for the service. The detection step is necessary to detect new feature conflicts and to give to the resolution step some helpful information. The detection step can use both off-line and on-line techniques to check an inconsistent service operation in the IN. Off-line detection techniques come mainly from verification and validation tools, simulation schemes of the environment, and the use of checklists. The number of services and service features involved, the number of ways in which features can interact, as well as the size of a complete, detailed feature 
implementation description will usually make the complexity of the off-line detection of all possible interactions too high to be feasible. Therefore, it is necessary to use on-line detection techniques to check the service process in the IN. Such techniques analyze the behavior of the service at different stages. The instantiated parameters and invoked features make easier the analysis of the interactions. The next sections present some of the main concepts used by the detection techniques.

\subsubsection{State-transition machines}

State-transition machines and detection algorithms can take part of an off-line automated solution, for the detection of feature interactions. In [Braithwaite 94] the service features are arranged in layers that represent the machines Each machine can pass its unaltered input, represented by a token, to the next machine and thus, the token is propagated through the layers. A call is represented by a single-ended call model based on agents rather than on connections.

An agent is a component of the system that participates in the establishment of a call. To avoid feature interaction problems, a state-transition machine is created for the different types of calls that the various features can modify. Finite-state machines are created to model the set-up and the release of the call. Then, features may be added on top of these machines. In that case, a detection technique is based on the execution of all possible configurations of the component feature machines on all possible call models. Some interactions are detected as a conflict between the required information for a certain state and the real information found in that state. Thus interactions can be detected by testing reachable states of a composite machine. A backtracking algorithm is used to build the reachability graph of the aggregation of the two features.

A similar solution using state-transition machines is proposed by [Ohta 94] where the approach takes into account the network from the user point of view, which is considered as a black box. Service specifications are given by the description of the behavior of the terminal. Interactions are modeled as unwanted properties (illegal or lost transitions) of these descriptions and a service specification language based on state transition rules is used to ensure this function. Building blocks can be also used to model features and their operating context. [Lin 94] suggest that only three operating contexts are enough to treat all relevant cases of interactions: originating side of the call, terminating side, and two-parties call. Again finite-state machines are used to obtain a procedural description of the feature behavior.

A composite technique is presented by [Gammelgaard 94]. It use Finite State Machines and logic to define labeled transition systems over which network properties (properties in all states) can hold transition rules. If a state fulfills a (first-order) precondition, a certain transition is enabled and the target state fulfills a corresponding post-condition. Feature interaction problems appear in that case when certain logical models are not possible. Network properties and pre and post conditions of declarative transition rules are formulated in a simple logic that is a restriction of an ordinary first order logic. Both rules are taken simultaneously into account. There is a detection of an interaction if some requirements on the resulting state are not satisfied. The advantage of using such a technique is the possibility to carry out standard logical deductions and to support them by explicit network properties. 
On the other hand [Inoue 92] and [Ohta 93] use non determinacy in the selection of transition rules to detect interactions; if two rules are both true in the global state and if a certain priority rule does not apply, there is an interaction. We can notice that the method of [Ohta 93] is well suited for the detection of interactions while the method of [Gammelgaard 94] deals with the interference [Mierop 92] or with the policy interaction [Gaarder 93], which are very closed concepts.

\subsubsection{Logic}

The concepts of constraints and knowledge goals [Halpern 90] are also used to detect feature interactions [Faci 94]. Conștraint-oriented specifications are developed in LOTOS [ISO 8807]. A type of operator corresponds to each type of constraints. The designer defines a set of knowledge goals and verifies their reachability when the involved features in the service process are active. If a goal is unreachable, the designer concludes that a feature interaction (or design error) exits. The goal is materialized by the behavior section in LOTOS process specifications.

[Combes 94] propose a user view abstract model using the SDL language [ITU-T Z.100]. The methodology is based on concepts coming from the Service Plane level of the INCM architecture [ITU-T Q.1205, 93] and from the temporal logic. The feature requirements are expressed as properties through a language based on a modal or a temporal logic. Interactions are detected when the system is not able to satisfy these properties [Combes 93].

Temporal logic [Manna 92] and Finite State Machine models are also used by [Blom 94] for an off-line detection of feature interactions. This logic coming from Lamport's Temporal logic of Actions [Lamport 91] is based on first-order formulas (with event predicates and actions describing the state-space changes) using the linear temporal operator always. Interaction in this case is characterized by an inconsistent system description resulting from the interaction behavior of two features.

\subsubsection{Design methodologies and creation environments}

Another way to decompose the system and to downsize the complexity of the interaction problem is to group together all the features which have similar functionalities.

[Kimbler 94a] suggest to break up the services into their different features and to concentrate the analysis on possible interactions between features which belong to categories subject to interactions in different stages of their life-cycle. A spotting algorithm is used to recognize combinations of features subject to interactions. This algorithm uses information about the interactions among the feature categories and the interactions among the services.

Use Case Driven Analysis (UCDA) is also proposed to detect combinations subject to interactions of service features [Mills 87]. The Use Case Driven Analysis is a widely spread technique using an object oriented software engineering [Jacobson 92], [Rumbaugh 91]. An approach based on UCDA is given by [Kimbler 94b]. The method starts from an informal user-oriented description of a service by generating scenarios described as sequences of events and user interactions with the system. These scenarios are transformed into service usage models describing the dynamic behavior of the system from the user point of view. 
The analysis aims to find different possible scenarios of the system operation. Several models are built during the analysis; the most important models are the User Case Model (UCM) and the Service Usage Model (SUM) [Kimbler 94a].

The User Case Model (UCM) describes Actors and User Cases. The Service Usage Model (SUM) contains dynamic models of services. A User Case is a specific scenario of the system operations described as a sequence of events and interactions between the user and the system. It is obvious that User Case Models will not cover all the possible combinations of services and features and all the exceptional situations that might happen during the call set-up and the release process. But those models look like a structure that helps the designer to analyze the functionalities of a complex system under different point of views.

[Mierop 92] also use an object oriented environment to assist the designer to model and to analyze the effects of the introduction of a new service and to solve service interactions (interferences). The concept of interaction is here quite different from its general meaning. A service interaction is not considered as a negative point in the service process. The interference concept tends to underline the unwanted aspect of an interaction in general.

An approach tries to avoid, to detect and to solve the service interaction (interference) in the Service Creation Environment (SCE). ROSA is an object oriented architecture composed of two separate architectural frameworks: the Service Specification Framework (SSF) and the Resource Specification Framework (RSF) [Oshinsanwo 92]. ROSA has developed an object model, the ROSA Object-Oriented Model (ROOM), which focuses on the description of services. This model is very similar to the ANSA object model [APM 91].

A service is viewed by a client as a set of operations (on encapsulated data). In this approach, an object is a group of interfaces (Service Provision Points) and the associated data. An object has multiple interfaces and the number of interfaces can change. An object can also be created dynamically. The approach relies on dynamic objects and on an interface creation to configure dynamically telecommunication services. Each object has a clearly defined responsibility in the system, which helps the designer to understand the implication when new objects are added or when some objects are changed. We notice that changing the internal behavior of an object will not generally change its role in the system.

The detection strategy is based on a special object called user profile which knows to which services a user is registered and under which conditions certain (supplementary) services can be activated.

\subsubsection{Other concepts}

On the other side, [Cameron 91] use the concept of the update-inconsistency as a condition to detect interactions. If, using the select-all policy, two features force some variables to be updated more than once in a cycle, then there is an interaction. [Cameron 91] use a single variable to record active features. This gives an update-inconsistency as soon as two features start up at the same event, even if the features are not in conflict.

[Tsang 94] suggest an on-line detection technique, based on the introduction of a Feature Manager (FM) that checks if an activated feature displays the correct behavior. The feature is required to behave as a deterministic Finite-State Machine could do. The FM should be able to perform a behavior analysis and an analysis of the resources modified by the feature at each 
step. The advantage is that we do not need a centralized knowledge. The FM as a part of the Feature Management System (FMS) represents a low-cost approach requiring no modifications on the $\mathbb{I N}$ architecture. When a feature is instantiated, a Feature Manager (FM) instantiation is also performed. This instantiation includes the storage of a copy of the sequences that monitor the features. These sequences are independent from the other FMs. The stored sequences stay in a central database and each FM monitors only one feature instantiation.

Each feature has a particular sequence of state transition called state signature, which represents the behavior of the feature. When the activated state sequence does not match with the sequence signature of the feature, an interaction problem has to be expected. It appears that using only state information is a disadvantage. The size and the number of sequences have to be limited. If not, the inclusion of data is expected to cause a state explosion.

We notice that off-line validation tools and simulation environments try to recreate the original and real environment of the IN as it is going to be tested with on-line approaches. Clearly these techniques cannot replace completely and efficiently the environment of the IN. Moreover, on-line detection approaches are limited to certain classes of interactions when a service is tested. It was shown that certain parts of these interaction situations can occur when very specific conditions are met. This makes that on-line detection techniques are not able to eliminate all possible causes of interactions. Then, it is necessary to solve these interactions when they occur.

\subsection{Resolution}

Once an interaction is detected, resolution techniques have to be engaged to eliminate this interaction or at least to minimize the effects on the environment. Off-line and on-line resolutions can be used in that case. Off-line approaches include the manual redesign or the reimplementation.

On line approaches try to find a way to solve an interaction when this interaction occurs or even when it has occurred, to try to minimize its consequences. Feature-interaction manager (FIM) in the IN, event-based resolution mechanisms and negotiation schemes are different approaches to solve the interaction.

[Cheng 94] presents a technique for the incremental service specification and for the interaction management concept to support the incremental specification and addition of services. This method is mainly based on the process composition specified in LOTOS [ISO 8807]. Each new service has to be specified independently using a common call model and/or an existing or a new Service/Service Feature (S/SF). Thus, the new service is developed by chaining Service/Service Feature behaviors together. The overall specification approach consists in a sequence of process behavior interconnected by links. The links establish a control and/or a data relationship (interaction) between two or more process behaviors.

A starting and a terminating process have to be defined when the different activities are chained. Constraints are applied during the process composition to manage and to solve interactions. LOTOS permits the introduction of such formal constraints on the parallel composition and on the synchronization of several processes. 
The appropriate resolution of the interaction requires to know the intention of the subscribers when they activated the features. Typically, a particular implementation of a feature does not represent the ultimate intention of a user, but just a way to achieve the intention of the customer. There might exist alternative ways to achieve the intention of the customer. [Griffeth 94] assume that constraints can be expressed in terms of operations that a user or a provider is willing or unwilling to perform. To solve conflicts between the constraints of different users, a mechanism of negotiation is provided to determine which set of operations will be used to initiate or to modify a call.

An important consequence of the use of the negotiation principle is that the autonomy of different users and providers can be preserved [Griffeth 93a], [Griffeth 93b]. It appears that to solve the interaction, one must favor one feature over another [Griffeth 94], introducing this way, a priority concept.

A negotiation mechanism must provide the user agent a way of exploring all the possible alternatives to achieve his intention. After receiving a request to set-up a call which is unacceptable for the user, the user agent must recognize which intention might be behind the request and then to derive from that intention alternative ways (possibly acceptable) to achieve it. Even when a user is not informed explicitly about the intention of other users, he may be able to speculate about the intention, based on the information he has, i.e., the received request.

The system is composed of three concepts which use negotiation: the platform, the negotiation objects and the user interface. The mechanism of negotiation assumes that a call initiation and a modification are described by a collection of operations that determine the form of a call. Policy feature and technology features are both related to these operations. The technology feature determines which operations are available and the policy feature determines which operation a system user is willing to use. Object-oriented platforms help to address a technology feature which is an individual operation that the platform provides. Thus, the feature can be added or removed rapidly and easily from the system.

A policy feature is a constraint on a set of operations that a user or a provider is willing to perform to initiate or to modify a call. This set of operations is sent to the negotiating system, which returns different sets of operations to be executed. The negotiation mechanism guarantees that the concerned users and providers have authorized any set of operations it returns.

This mechanism works on behalf of entities, which have policies to select operations they want to perform. Agent objects represent the various entities of the system and try to carry out their policies. Negotiators help the agent to reach an agreement.

An agent-object is assigned to each entity in the network. The functions of an agent are to produce proposals, to initiate or to modify a call, to evaluate proposals coming from other agents, and to generate counter-proposals. The proposals have to specify the desired operations of the calls. If a proposal is not acceptable to one entity, the agent constructs a counter-proposal.

Three ways of negotiation exist [Griffeth 94] :

- Direct negotiation, where the agent negotiates directly with the other agents without the assistance of a mediator. 
- Indirect negotiation, where a monitor called negotiator is used for the progress of the negotiation. The negotiator is a dedicated entity that recognizes which agent has to approve a proposal and routes proposals and counter-proposals to the appropriate agents.

- Arbitrated negotiation, where the agents do not need to generate and to evaluate proposals.

An arbitrator takes the complete script of each agent and has the responsibility to find a resolution of the conflict.

The use of a negotiation object permits to avoid some problems as:

- Non terminating sequence of proposals and counter-proposals;

- The introduction of new agents, and the facilities to communicate with them;

- Negotiation can store knowledge and use it for further conflicts (experience);

- Different mediators can be used for different situations providing benefits of specialization (smart or dumb negotiators).

The mediator is supposed to find an agreement that should be quite good for all parties.

The user interface allows the user and the service provider to access two kinds of functionality: to express constraints and to submit proposals. These constraints can be expressed in any logic languages. The basis of the mechanisms necessary for the negotiation is to provide a goals hierarchy, where the lowest level (basic) is characterized by operations on a given platform and where the higher level corresponds to the possible goals or intentions of the users. New goals can be defined by combining other goals. A goal is realized when the combination of the sub-goals leading to a final goal is achieved.

\section{CONCLUDING REMARKS}

A global solution for the interaction problem will probably use many concepts and solutions from different research fields. At a logical level, we trust that this solution will use objectoriented concepts, associated with a strong formalism to build a representative model of the IN system. The object methods for the analysis and the conception of object-oriented systems will probably also have an important role to play for the design of such telecommunication systems.

The dynamic operations of the system will probably be controlled by Finite State Machines associated with logical rules of transition. State signature, object profile or feature manager concepts may also constitute good strategies to detect feature interactions. Even if we take care of the two first stages, avoidance and detection, the resolution stage will always be necessary due to the limitation of simulation tools to approach the real environment and the operations performed in the $\mathbb{I N}$. Negotiation techniques seem to be an efficient solution for the real time and for the autonomous resolution of interaction problems. The problem with these techniques is first, to know : $f$ the search of an interaction solution will not take too much time and second, if such an autonomous system will not perform an unexpected behavior. 


\section{REFERENCES}

[APM 91] An Application Programmers Introduction to the Architecture, Release TR.017.00, APM Ltd, 1991.

[Arango 92] M. Arango et al., Touring Machine: A software Infrastructure to Support Multimedia Communications, Multimedia'92, 4th IEEE COMSAC Int'I, Wksp. on Multimedia Communications, Monterey, California, April 1-4 1992.

[Bendeich 93] J. Bendeich, I. Black, C. James, J. Ladmore and P. Sgangarella, Advanced Telecommunications services : MORGAN, User and Technical Manuals, 1993.

[Blom 94] J. Blom, B. Jonsson and L. Kempe, Using Temporal Logic for Modular Specification of Telephone Services, Feature Interactions in Telecommunications Systems, pp. 197-215, IOS Press, Amsterdam, May 1994.

[Bouma 94] W. Bouma and $\mathrm{H}$. Velthuijsen, Introduction, Feature Interactions in Telecommunications Systems, pp. VI-XIV, IOS Press, Amsterdam, May 1994.

[Bowen 89] T.F. Bowen, F.S. Dworack, C.H. Chow, N.Griffeth, G.E. Herman and Y.J. Lin, The feature interaction problem in telecommunications system, SETS, 1989.

[Bowen 90] T.F. Bowen, C. Chow, F.S Dworak, N. Griffeth and Y. Lin, Views on the Feature Interaction problem, Manuscript, Morristown, Bellcore, 1990.

[Braithwaite 94] H. K. Braithwaite and M. J. Altee, Toward automated Detection of Feature Interactions, Feature Interactions in Telecommunications Systems, pp. 258-259, IOS Press, Amsterdam, May 1994.

[Brooks 87] F. Brooks, No Silver Bullet, Essence and Accidents of Software Engineering, IEEE Computer, vol 20(4), pp. 10-19, April 1987

[Cain 92] M. Cain, Managing Run-Time Interactions Between Call-Processing Features, IEEE Communications Magazine, pp. 44-50, February 1992.

[Cameron 91] E.J. Cameron and Y.J. Lin, A Real Time Transition Model for Analysing Behavioral compatibility of Telecommunication services, proceedings of the ACM SIGSOFT'91, Conference on software for Critical Systems, pp. 101-111, ACM Press, December 1991.

[Cameron 93] E. J. Cameron and H. Velthuijsen, Feature Interactions in Telecommunications Systems, IEEE Communication Magazine, August 1993.

[Cameron 94] E.J. Cameron, N.D. Griffeth, Y.J. Lin, M.E. Nilson, W.K. Schnure and H.Velthuijsen, A Feature Interaction benchmark for IN and beyond, Feature Interactions in Telecommunications Systems, pp. 1-23, IOS Press, Amsterdam, May 1994.

[Cheng 94] K. E. Cheng, Toward a Formal Model for Incremental service Specification and Interaction Management Support, Feature Interactions in Telecommunications Systems, pp. 152-166, IOS Press, Amsterdam, May, 1994.

[Combes 93] P. Combes, B. Renard,W. Bouma and H. Velthuijsen, Formalisation of properties for feature interaction detection, International Conference on Intelligence in Service and Networks, Paris, France, November 1993.

[Combes 94] P. Combes and S. Pickin, Formalisation of a user View of Network and Services for Feature Interaction Detection, Feature Interaction in Telecommunications Systems, pp. 120-135, IOS Press, Amsterdam, May, 1994. 
[Cross 94] M. Cross and F.O'Brien, Restructuring the Problem of Feature Interaction : Has the approach been Validated ? Experience with an Advanced Telecommunication for Personal Mobility, Feature Interactions in Telecommunications Systems, pp. 249-257, IOS Press, Amsterdam, May 1994.

[Dankel 94] D. D. Dankel II, K. Nielsen, M. Schmalz, L. Muzzi, W. Walker and D. Rhodes, An Architecture for Defining Features and Exploring Interactions, Feature Interactions in Telecommunications Systems, pp. 258-259, Amsterdam, IOS Press, May 1994.

[Erradi 92] M.Erradi, F. Khendek, R. Dssouli and G.Bochmann, Dynamic extension of objectoriented distributed system specifications, International Workshop on Feature Interactions in Telecommunications Software Systems, pp.116-132, St. Petersburg, Florida, USA, December 3-4 1992.

[Faci 94] M. Faci. and L. Logrippo, Specifying Feature and Analysing Their Interactions in a LOTOS Environment, Feature Interaction in Telecommunications Systems, pp. 136-151, Amsterdam, IOS Press, May 1994.

[Gaarder 93] K. Gaarder and J. Audestad, Feature interaction policies and the undecidability of a general feature interaction problem, TINA'93, pp. II-189-II-200, 1993.

[Gammelgaard 94] A. Gammelgaard. and J. Kristensen, Interaction detection a logical approach, Feature Interaction in Telecommunications Systems, pp. 178-195, Amsterdam, IOS Press, May 1994.

[Griffeth 93a] N. D. Griffeth and H. Velthuijsen, Reasoning about goals to resolve conflicts, Proceedings International Conference on Intelligent Cooperating Information Systems (ICICIS-93), pp. 197-204, Rotterdam, IEEE Computer Society Press, May 12-14 1993.

[Griffeth 93b] N. D. Griffeth and H. Velthuijsen, Win/ win negotiation among autonomous agents, proceedings 12th International Workshop on Distributed Artificial Intelligence, pp. 187-202, Hidden Valley, PA, May 19-21 1993.

[Griffeth 94] N.D. Griffeth and H. Velthuijsen, The negotiation Agent Approach to Runtime Feature Interaction Resolution, Feature Interaction in Telecommunications Systems, pp. 217-235, Amsterdam, IOS Press, May 1994.

[Halpern 90] J.Y. Halpern and Y. Moses, Knowledge and common knowledge in a distributed environment, Journal of the ACM, 37(3), pp.549-587, July, 1990.

[Harel 92] D. Harel, Biting the Silver Bullet, IEEE Computer, Vol 25(1), pp. 8-20, January 1992.

[Herbert 93] A. Herbert, Personal Comment at the Feature Interaction Session, TINA'93, L'Aquila, Rome, September 27-30 1993.

[Hopcroft 79] J.E. Hopcroft and J.D. Ullman, Introduction to Automata Theory, Languages and Computation, Addison-Wesley, 1979.

[IEEE 93a] IEEE Communication Magazine, Special issue on IN, March 1993.

[IEEE 93b] IEEE Communication Magazine, Special issue on Feature Interaction, August 1993.

[IEEE 93c] IEEE Computer, Special issue on Feature Interaction, August 1993.

[Inoue 92] Y. Inoue, K. Takami and T. Ohta, Method for supporting detection and elimination of feature interaction in a telecommunication system, International Workshop on feature 
Interaction in Telecommunications Software Systems, pp. 61-81, IEEE Communication Society, December 1992.

[ISO 8807] ISO, LOTOS, A Formal Description Technique Based on the temporal Ordering of Observational Behavior, Information Processing Systems, Open Systems Interconnection, International Standard IS 8807, ISO, 1989.

[ISO 9074] ISO, ESTELLE, A Formal Description Technique Based on Extended State Transition Model, Information Processing Systems - Open Systems Interconnection, ISO/TC 97/SC 21, ISO 9074, 1989.

[ISO/IEC 10746] ISO, ITU-TS, Recommendation X.9000: Basic reference Model of Open Distributed Processing, Draft International Standard, ISO/IEC 10746, March 1994.

[ITU-T Q.12xx 93] ITU-T, Recommendations Q.120x and Q.121x, 1993.

[ITU-T Q.1203, 93] ITU-T, IN Global Functional Plane Architecture, Recommendation text Q. 1203, 1993.

[ITU-T Q.1204, 94] ITU-T, IN Distributed Functional Plane Architecture, Recommendation text Q. 1204, 1994.

[ITU-T Q. 1205, 93] ITU-T, IN Physical Plane Architecture, Recommendation text Q. 1205, ITU-T, 1993.

[ITU-T Q. 1211, 92] ITU-T, Q.1211 Recommendation for Target Services and Services Features for IN CS-1, 1992.

[ITU-T Z.100] ITU-T, Specification and Description Language SDL, Recommendation Z.100, 1987.

[Jacobson 92] I. Jacobson et al., Object-Oriented Engineering, A Use Case Driven Approach, Addison-Wesley, 1992.

[Lin 94] F. J. Lin and Y.J. Lin, A building block Approach to Detecting and Resolving Feature Interactions, Feature Interactions in Telecommunications Systems, pp. 86-119, Amsterdam, IOS Press, May 1994.

[Kimbler 94a] K. Kimbler, E. Kuisch and J. Muller, Feature Interactions among Pan-European Services, Feature Interactions in Telecommunications Systems, pp. 73-85, Amsterdam, IOS Press, May 1994.

[Kimbler 94b] K. Kimbler and D. Söbrik, Use Case Driven Analysis of Feature Interactions, Feature Interactions in Telecommunications Systems, pp. 167-177, Amsterdam, IOS Press, May 1994.

[Lamport 91] L. Lamport, The temporal logic of actions, Technical report, DEC/SRC, 1991.

[Linden 94] R. van der Linden, Using an Architecture to Help Beat Feature Interaction, Feature Interactions in Telecommunications Systems, pp. 24-35, Amsterdam, IOS Press, May 1994.

[Manna 92] Z. Manna and A. Pnueli, The Temporal Logic of Reactive and Concurrent Systems, Springer Verlag, 1992.

[Mierop 92] J. Mierop, S. Tax and R. Janmaat, Service Interaction in an Object Oriented Environment, International Workshop on Feature Interactions in Telecommunications Software Systems, pp. 133-152, St. Petersburg, Florida, USA, December 3-4 1992.

[Mills 87] H. Mills et al., Cleanroom Software Engineering, IEEE Software, September 1987. 
[NT 89] Northern Telecom, Northern Telecom Software, 5204.11/11-89, Issue 1, Northern Telecom, Research Triangle Park, NC, 1989.

[Ohta 93] T. Ohta, K. Takami and A. Takura, Acquisition of service specifications in two stages and detection/resolution of feature interactions, Tina' 93, pp. II: 173-187, September 1993.

[Ohta 94] T. Ohta and Y.Harada, Classification and Resolution of Service Interactions in Telecommunication Services, Feature Interactions in Telecommunications Systems, pp. 60-72, Amsterdam, IOS Press, May 1994.

[OMG 94] OMG, The Common Object Request Broker: Architecture and Specification, Revision 1.1, OMG Document number 91.12.1, 1991, Revision 1.2, OMG, 1994.

[Oshinsanwo 92] A. O. Oshinsanwo et al., The RACE Open Service Architecture Project, IBM Sys. J., vol 31, no 4, December 1992.

[Rumbaugh 91] J. Rumbaugh et al., Object-Oriented Modeling and Design, Prentice Hall, 1991.

[TINA 92] Proc. 3rd Wksp. on Telecommunication Network architecture (TINA), Narita, Japan, January 1992.

[Tsang 94] S. Tsang and E.H. Magill, Detecting Feature Interaction in the Intelligent Network, Feature Interaction in Telecommunications Systems, pp. 236-247, Amsterdam, IOS Press, May 1994.

\section{BIOGRAPHY}

Dominique Gaiti received the Ph.D. and the habilitation degrees in Computer Science from the University of Paris VI and Paris IX, France, in 1991 and 1995, respectively. She is currently an Associate Research Scientist at the Center for Telecommunications Research (CTR), Columbia University, New York, after two years spent as a Visiting Scientist in the same lab. She also works at the University of Paris VI, France, as a member of the Scientific Staff. Her research interests are in network management and control, intelligent networks and distributed artificial intelligence.

Nadir Belarbi was graduated in 1993 as a computer science engineer from the University of science and technology, Oran, Algeria. He obtained a Master degree (DEA) in computer science and telecommunications from the University of Paris $\mathrm{V}$ and the Ecole Nationale Superieure des Telecommunications (ENST), Paris, France. He currently prepares a Ph.D. degree from the University of Versailles, France. His research interests include management of the service interaction in Intelligent Networks (IN), Artificial Intelligence and distributed systems. 